\title{
Therapeutic HPV vaccine holds promise
}

Prophylactic human papillomavirus (HPV) vaccination prevents cervical intraepithelial neoplasia (CIN) caused by high-risk HPV strains (which include HPV types 16 and 18 that cause $70 \%$ of cervical cancers), but have no therapeutic effect on established lesions. Now, a DNA vaccine, VGX-3100, which targets the HPV16/18 E6 and E7 oncoproteins, has shown therapeutic efficacy in 167 women with biopsy-proven HPV16/18-associated CIN2/3 in a phase II trial.

The women were randomly assigned 3:1 to receive VGX-3100 or placebo, and all underwent standard-of-care resection of the cervical squamocolumnar junction 24 weeks after their third vaccination. "Nearly half of the patients vaccinated ( 49\%) had histological lesion regression," states lead author Cornelia Trimble. In both per-protocol and intention-totreat analyses, the frequency of lesion regression was $\sim 19 \%$ higher in the vaccine cohort versus placebo cohort $(P=0.034$ in both analyses). "Vaccinated patients whose lesions regressed were much more likely to have concomitant viral clearance (80\% versus $50 \%$ ), and were twice as likely to regress to normal, compared with unvaccinated patients, who were more likely to regress to CIN1," adds Trimble.

Vaccination with VGX-3100 elicited robust $\mathrm{T}$-cell and antibody responses to the E6/E7 antigens; as Trimble explains, "this contrasts with other therapeutic DNA vaccines tested to date, and is probably related to the fact that VGX-3100 is administered using electroporation."

A phase III trial in women with HPV16/18-associated CIN2/3 is planned; clinical trials of VGX-3100 in other $\mathrm{HPV}$-associated cancers are ongoing.

\section{David Killock}

Original article Trimble, C. L. et al. Safety, efficacy, and immunogenicity of VGX-3100, a therapeutic synthetic DNA vaccine targeting human papillomavirus 16 and $18 \mathrm{E} 6$ and E7 proteins for cervical intraepithelial neoplasia 2/3: a randomised, double-blind, placebo-controlled phase $2 \mathrm{~b}$ trial. Lancet doi:10.1016/S0140-6736(15)00239-1 